\title{
Optimizing E-Learning Cognitive Ergonomics Based on Structural Analysis of Dynamic Responses
}

\author{
https://doi.org/10.3991/ijet.v14i10.10134
}

\author{
Henda Chorfi Ouertani ${ }^{\bowtie}$, Ghada Alhudhud \\ King Saud University, Riyadh, Kingdom Of Saudi Arabia \\ houertani@ksu.edu.sa
}

\begin{abstract}
Smart Assistive Technologies can be a powerful tool in supporting education environments and inclusion for learners with visual/hearing impairments. For example, while captions in videos are a necessity for deaf users, audio reading is inevitable for blind ones. Including such technologies into a smart e-learning environment provide huge opportunities to customize the content presentation to needs and ability of learners. Despite the number of models being introduced during the last decade, acceptance model and behavioral model are, yet, exhibiting design drawbacks for learners with visual and hearing impairments. Meanwhile, the e-learning initiatives in the universities have paid great efforts in order to optimize usability of conventional e-learning systems. However, optimizing assistive e-learning systems is not covered in the recent research. Central to e-learning optimization is the learners' realization problem; in terms of the size of gap between learners' expectations and real interaction measures. This paper presents a study of measure the usability of assistive elearning systems and modeling better interaction based on adjusted Fitt's Law to consider time of movement for assistive technologies embedded in e-learning systems. The proposed usability evaluation considers the hardness of mental operations during e-learning various activities.
\end{abstract}

Keywords—Smart e-learning, usability evaluation, Fitt's Law adjustment

\section{Introduction}

Although there is a number of theories and models emphasizing the behavior of an individual towards using information technology, yet, little is known on the individual's experience while interacting with that technology [15]. The dissimilarity between design model of e-learning system and the real situation of the system implementation expressed what is known as a realization problem. In one hand, the design model of elearning system contains the designed assumptions and conceptions reflecting the user expectations. On the other hand, the real situation of the system implementation is represented by the learner experience. The existing e-learning realization problem creates the desire for extending the research about the effects of the cognitive dimensions and beliefs of the user while using the system. 
Expressing dynamic responses of the adaptive e-learning system concerns mainly with the adaptive interactions that take place at the systems interface. Adaptive interactions are intended for smart learners' interaction with the system, without modifying the learning content itself [21]. This adaptive interaction accommodates learners' impairment type. For example, some people with visual or hearing impairments have to use some kind of assistive technologies to access e-learning content. Each type of impairment requires certain types of adaptations in the design of e-content. For example, while captions in videos are a necessity for deaf users, audio reading is inevitable for blind ones. Including such technologies into a smart e-learning environment, provide huge opportunities to customize the content to needs and ability of learners. In this paper, we propose a usability study of a Smart Assistive Learning Technology (SALT) system. Based on the user profile that includes the disability type, SALT determines suitable tools and materials for disable learners. A number of learning activities and the corresponding learning style for each activity are defined. SALT system identifies and proposes the supporting learning styles for each commonly known disability. Experimentation results have been shown the system to be efficient for recognizing learner's single disability or multiple disabilities where applicable and identify the associated assistive technologies. This adaptive interaction would in turn reduce the hardness of mental operations for learners in recognizing the actions to be selected in the system.

\section{Theoretical Background}

\subsection{Behavioral model}

Dynamic responses concerns, in system design, are keystones in building a behavioral model for the human interaction with system elements. Applying these factors in system design and theories for optimum interaction is currently known as Cognitive Ergonomics (CE). Despite the high level technology in the current e-learning systems, there are some lack of interaction and low retention behavior reflect the learners experience while using the system. Hence, CEs are considered mainstays for building a design which supports optimum learner-system interactions.

Modeling the CEs in system design has been around a while ago. For example, Fitts Law [9] models the ergonomics prediction of the time required to move to a target area as a function of the distance and the size of the target. Another example is the Cognitive Dimensions framework [12]. It provides a simple and quick means of describing interaction scenarios. The Cognitive Dimensions framework classifies interactive systems from the perspective of the real practitioners using these systems. This makes classification more relevant to the practitioners in that they know and understand the strengths and weaknesses of the particular notation they are using. Yet models related to cognitive dimensions never addressed the specifics of e-learning systems.

The scope of this paper tackles the mental processes in the form of perception, responses as the CEs of interest. This is due to the fact that these factors affect the inter- 
actions among humans and other elements of a system and influence the mental workload, decision-making, skilled performance. The rest of the paper is organized as follows: theoretical background section provides information about the cognitive dimensions and the cognitive absorption theories [20] also the learnability dimension is provided. Next, a section presents the exploratory study to verify the relationship between hardness of mental operations and the use of embedded assistive technologies in the e-learning system. An experiment is described comparing the performance of an eye tracker and a mouse in a simple pointing task. Subjects had to make rapid and accurate horizontal movements to targets that were vertical ribbons located at various distances from the cursor's starting position. The dwell-time protocol was used for the eye tracker to make selections. Movement times were shorter for the mouse than for the eye tracker. Fitts' Law model was shown to predict movement times using both interaction techniques equally well. The model is thus seen to be a potential contributor to design the modern multimodal human-computer interfaces.

\subsection{Fitts law and e-learning}

Fitts's law has been introduced in [9]. Fitts' Law has been formulated mathematically in several different ways. The most common form is the Shannon formulation. Fitts law predicts the mean movement time incorporating the start/stop time of the device and the inherent speed of the device, and the width of the target measured along the axis of motion. Fitts' Law is used in e-learning as a model because of the similarity in activities related to learning and dimensions measured by the law. It emphasizes certain physical aspects of user's interaction with the system. However, the validity of Fitts' Law is limited because it does not address:

- Factors related to task completion times. The completion times are reduced when a task is split over two hands [6].

- Effect of human body performance difference between preferred and non-preferred hands [13], and the observation that subjects were uniformly more accurate in arm motions towards the body than away from the body movements.

- Drawing, writing, and steering activities, which are becoming common tasks in modern computer interfaces. New laws for this type of movements are highly demanded [1] and [18].

- Inclusion of assistive technologies in e-learning systems.

In summary, Fitts' Law supports low level activities, but not intermediate and high-level activities. The high level activities take the form of system response time, mental preparation time for the user, timing for selection rules for alternative methods, etc. [14]. These intermediate and high-level activities have been addressed in the Keystroke-Level Model (KLM) [17] and GOMS model [4], [5]. The KLM and GOMS are both concerned in pure dynamic factors effect on user responses on systems but not the cognitive effects of system responses on users. 


\subsection{E-learning and cognitive theories}

Cognitive theory is based on cognitive dimensions that affect user behavioral interaction within the dynamic and interactive system. Using cognitive dimensions is an analytical tool that focuses on the process of interaction, rather than on static analysis of artifacts, is being introduced in [11]. Authors introduced a technique for analytical information seeking, including programming languages, device interfaces, and interactive software user interfaces. Meanwhile, cognitive aspects of programming and similar activities are used for meta-analysis that looks at structural aspects of the system and identifies characteristics that may embed or enable certain kinds of interactions with the system [12], and in the form of instructions and descriptions [11]. Researchers in the field have addressed the lack of knowledge on the individuals' experience while using the technology. However, theories that can predict the determinant factors of technology acceptance do exist. For example, the Acceptance model, introduced in [15], is based on one of these theories focusing on the behavior of an individual towards using technology but not during using technology.

One may ask why Cognitive Dimensions and e-learning. In [12], authors have introduced the importance of the learner role as a user in participation in the learning evaluation process. As stated in [12], cognitive dimension approach is applicable in both interactive and static systems. In this context, authors in [16] focus on the interactive artifacts including the software environments. In addition, learning with computer simulations is characterized as exploratory learning [10], so that the learners have to discover general rules, procedures, or higher order skills reflecting an active attitude of the learner towards merging the information into the learners' cognitive structure.

Considering information technology non-specialists, studying the cognitive dimensions related to users' experience while using technology covers a wide range of very different dimensions in the learners' cognitive structure. This cognitive structure should be seen as a complementary to other approaches, e.g. heuristic and key stroke level models. This is quite important to avoid the results of poorly usable systems in terms of hardness to learn.

Conventional employment of cognitive dimensions' approach is considered efficient and superior to other approaches as it identifies activity types and it can be applied at any stage of design. Because they make sense to ordinary users as well as to specialists, this approach is based on thirteen different dimensions, each of which is cognitively relevant, giving a suitability profile for each dimension [12]. The following subsections include the list of cognitive dimensions and their short descriptions [3].

\subsection{The cognitive factors influencing hardness mental operation vs usability}

Hardness mental operation is a measure of how difficult is to comprehend [11]. Hardness of Mental Operations (HMOs) is mainly concerned with mental operations that exploit a high level of users' attention. This is quite important as it raised the usability problems [19], especially when a user may not have the right background 
knowledge to perform the operation. In other words, hard mental operations describe the need for thinking situation that reflect the complexity of reading or understanding or even worries regarding conforming to ethical issues related to the e-learning system while performing activities using the interactive system. The cognitive structure required for our study is listed below.

- Premature commitment: constraints on the order of doing things. The learner commits a certain sequence of actions to accomplish a goal while different efficient sequence of actions is performed in order to accomplish the same goal.

- Viscosity: resistance to change: Resistance to change measure the effort required to make a single change while using the interactive system notations or assesses a designs resistance to change [11]. High viscosity indicates that the user goes through a series of menus or web application or boxes to switch between two different states throughout content of the system. Reducing viscosity requires much effort to make a change. The worst scenario occurs if the user is aware about the consequences when choosing actions whilst it is the difficult to undo these actions upon realizing additional information. Viscosity commonly occurs with high premature commitment.

- Role-expressiveness: this dimension is about how well the interface components reflect their purpose; the operations available on them, e.g. quick search components, copy and paste.

- Error-proneness: Indicates the ability where the user may find it easy to fall in error or unexpected mistake with little protection [7] and [2]. The level of tolerance and recovery the system can support for inevitable mistakes.

Hard mental operations highly require cognitive resources. Accordingly, evaluating the number of required cognitive resources in assistive e-learning systems constitutes the main goal of this study. This requires reviewing the set of the cognitive factors and trades-offs between these various factors in terms of the learner's expectations. For example, in order to reduce the hardness of mental operations during interaction, it is vital to reduce error proneness; i.e. how easy the user find it to fall in error or unexpected mistake and gets over it [7], [12]. Reducing the hardness of mental operations can also be achieved by minimizing the effect of premature commitment and viscosity. In other words, this can be viewed as reducing the difficulties the user may experience knowing what to do or what to undo when an error or unexpected result is observed [11]. Meanwhile, reducing the hardness of mental operations is also associated with improving both the role expressiveness and Consistency [7].

\subsection{Cognitive factors influencing learnability}

Measuring the difficulty in learning how to use the system is a key indicator of system' usability and is considered quite important for interactions with e-learning systems [8]. Learners in most cases are mainly concerned with the better recognition of the interface components like icons buttons and menus representing the components 
of the interface. This implies the: a) the perception of system responses, b) the consistency and c) expressiveness of the icons roles.

Perception is an important factor and indicates the ability and easiness to perceive the interface components easily. As an example, a user encounters a deep menu system. For learners with visual impairments, a poor visibility factor would affect the mental operations because the user needs to recall the step by step to perform a specific task. Accordingly, the learner experiences hardness of remembering the hierarchy while navigating this deep menu in order to discover the right option, to select and use. In addition, visibility reflects how easy it is to view the progress of system performance whilst performing a task. For example, when a learner tries to upload a file, it is quite crucial for the user to visualize the progress of the upload process. Another factor is the consistency. This is a measure of the degree of similarity in terms of the means of performing similar tasks in different parts of the interface. It applies to layout and to the availability of features. Role-expressiveness is another cognitive factor and is considered as a key component of the cognitive structure that heavily impacts the learnability. Role expressiveness indicates the purpose of each entity composing the interface of e-learning systems.

\section{Pilot Experimentation}

The system was tested for 120 users. Each of the 120 users defined disability type(s). 70 users were defined as single disability, whilst 50 users are defined as with multiple disabilities. The experiment was run in the form of a questionnaire in order to capture the learners' expectations regarding the hardness of mental operations, level of usability and tolerance by the system for learners' errors and the number of encountered technical problems. The subjects were asked to complete a task such as uploading an assignment, checking the calendar, converting media file, accessing project forum, and/or downloading a document. A comparison experiment is conducted to compare between the students mental processing time (using the proposed technology and without the proposed technology) in order to complete a task such as uploading an assignment, checking the calendar, converting media file, accessing project forum, and/or downloading a document, please, refer to Table 1.

The results of surveying the learners' expectations regarding hardness of mental operations they encountered whilst using the assistive e-learning system are presented. These expectations express the economics of cognitive exploitation related to mental operation, responses while using the e-learning system. The economics based expectations are shown in the plots in Figure 1. The top plots in Figure 1 demonstrate the summary of beginners' expectations, from left to right, in terms of the HMOs. The middle plots in Figure 1 demonstrate the summary of middle experienced learners' expectations, from left to right, in terms of the HMOs. At beginners and middle levels of expertise, about $69 \%$ of e-learners expect less number of training sessions needed, less encountered technical problems, less number of connectivity and accessibility problems as well as availability anytime. The bottom plots in Figure 1 demonstrate the summary of experts' expectations, from left to right, in terms of the HMOs. For 
the expert learners 97\% expect less HMOs, less technical problems, and wide connectivity options. By taking into consideration the learners, the interaction rate increase. Hence, by increasing the interaction rate the learners continue using the system after passing a few training sessions.
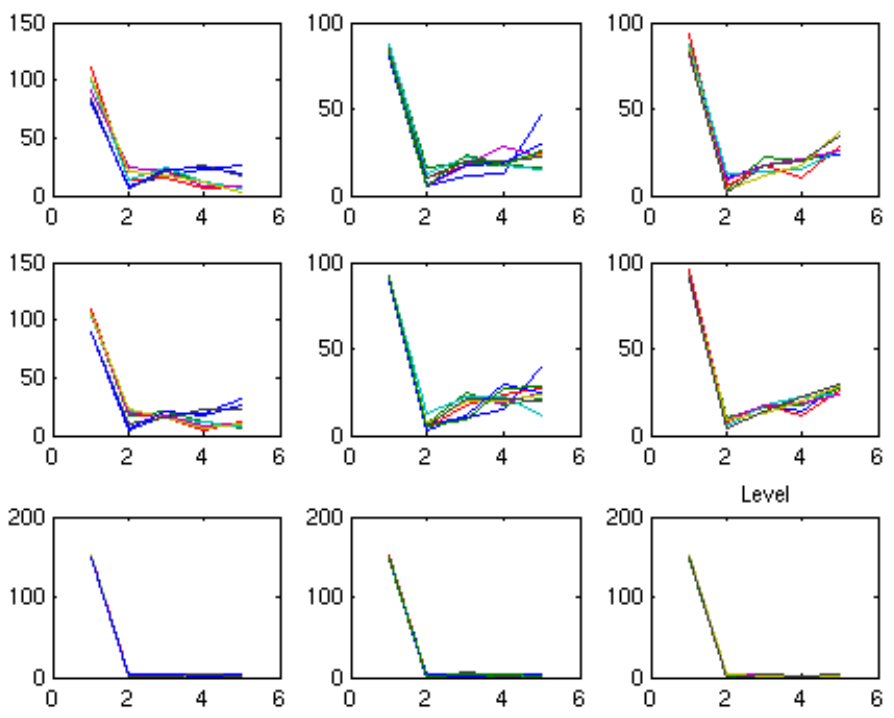

Fig. 1. Developing frequencies from beginners' level to experts' level.

A comparison experiment is conducted to compare between the students mental processing time (using the proposed technology and without the proposed technology) in order to complete a task such as uploading an assignment, checking the calendar, converting media file, accessing project forum, and/or downloading a document, please, refer to Table 1 . The average mental processing time has dropped by $30 \%$ using the assistive e-learning system over the regular e-learning system, Figure 2.

Table 1. Visually impaired Tasks Duration in seconds with and without the assistive technology

\begin{tabular}{|l|c|c|c|}
\hline \multicolumn{1}{|c|}{ Activity Type } & \# of participant & $\begin{array}{c}\text { Users of non-assistive } \\
\text { technology }\end{array}$ & $\begin{array}{c}\text { Users of Assistive learn- } \\
\text { ing }\end{array}$ \\
\hline Uploading an assignment & 20 & 4 & 3 \\
\hline Checking calendar & 20 & 5 & 3 \\
\hline Checking new slides & 20 & & 2.57 \\
\hline Converting media & 20 & 12 & 7.9 \\
\hline Accessing project forum & 20 & 3.8 & 2.5 \\
\hline Downloading content & 20 & 7.6 & 10.4 \\
\hline
\end{tabular}




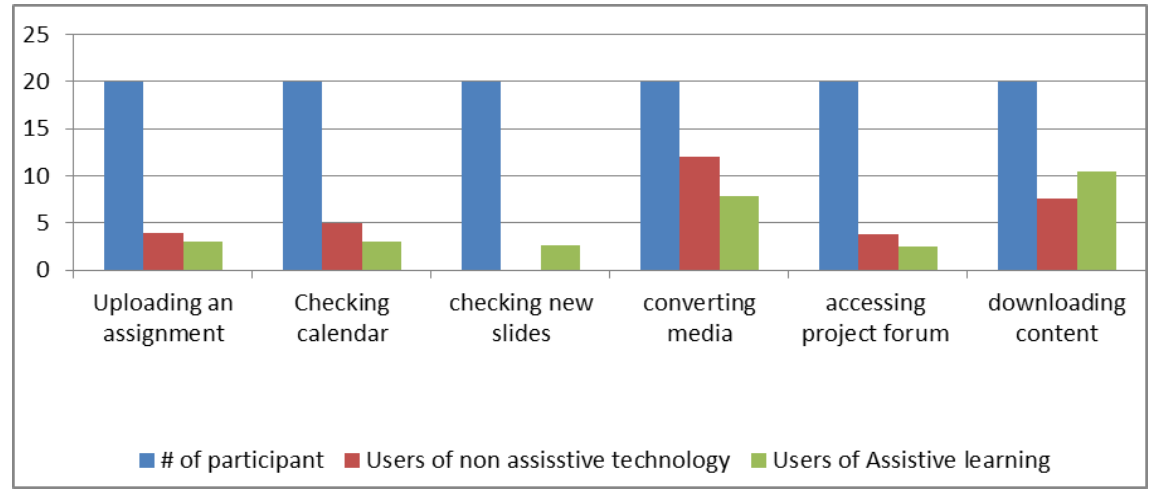

Fig. 2. Mental processing time for users of assistive technology in LMS and users of LMS without assistive technology

\subsection{The proposed behavioral modeling of usability of assistive e-Learning system}

Inspired by the trade-offs presented in pilot study in the previous section, the paper proposes and introduces economic modeling of cognitives in use during user interaction with assistive e-learning system. The proposed cognitive structure would ensure a reduction of the cognitive factors affecting hardness of mental operations and significant increase of the usability factors for better interaction with any e-learning system. Aspirations of the proposed model are to improve the degree of learnability through the system by reducing the probability of facing hard mental operation situation would be with great effect on reducing the resistance to change status. Additional effort is being devoted to reduce the effects of other dimensions that allows for activity that may involve hard mental operation situation such as (premature commitment, viscosity, error-proneness), and increasing at the same time the effects of usability (consistency, visibility and the balanced in designing the role-expressiveness dimension). This would guarantee pushing the user towards the absorption stage [20] while interacting with the e-learning system. The cognitive dimensions affecting e-Learners' mental operations contribute to the learnability. Hardness of Mental Operation (HMO) can be expressed in term of premature commitment $P_{c}$, viscosity Vis, errorproneness $E_{p}$

$$
\begin{gathered}
H M O \alpha V i s \\
H M O \alpha P_{c} \\
H M O \alpha E_{p} \\
H M O=V i s * P_{c} * E_{p}
\end{gathered}
$$

At the same time, usability can be increased if the e-learning system is highly learnable. Increasing learnability implies providing consistency $C$, visibility $V$ is and 
the role-expressiveness $R_{e}$. In this context, learnability can be mathematically expressed in terms of these factors as follows:

learnability $\alpha C(5)$

$$
\begin{gathered}
\text { Learnability } \alpha V \\
\text { Learnability } \alpha R_{e} \\
\text { Learnability } \alpha C * V * R_{e}
\end{gathered}
$$

According to relationships above, learnability can be expressed as how quickly and comfortably a new user can begin efficient and error-free interaction with the system. Incorporating the equations 4,8 would guarantee high usability [15], while interacting with the e-learning system.

$$
\text { Usability }=\frac{\text { learnability }}{H M O}
$$

\section{Conclusion}

Learners with visual or hearing impairments can benefit from the capabilities of an Adaptive Assistive Learning Environment in different ways. Those capabilities are nowadays benefiting from the rapid development of assistive technologies. Appropriate use of assistive technology in an adaptive learning environment enables all learners to experience learning activities regardless their differences or disabilities. Based on the user profile that includes the disability type, a number of defined learning activities are proposed. The usability study in this paper is composed of two parts. The first part of the experiment was a pilot study aimed at classifying and identifying the learner's expectations in the form of surveying the cognitive factors affecting the level of interaction with e-learning system. Examples for these factors are premature commitment, viscosity, and error-proneness. These are classified as factors lead to hardness of mental operations. Hence, the study considered the effect of increasing the learnability degree by providing consistency, visibility and the balanced in designing the role-expressiveness dimensions. Next stage is to measure the real interaction results in term of time records for performing tasks including different activities. In this phase, the set of designed experiments aims at comparing the predictions related to these expectations and the behavior of e-learners' and system performance during interacting with technology. The primary goal is the computation of the hardness of mental operations required for performing a task. Analytical view to compare times required for performing a task includes accurate analysis that examines the dynamic response.

\section{$5 \quad$ References}

[1] J. Accot and S. Zhai. Beyond fitts' law: Models for trajectory-based hci tasks. In Proceedings of CHI '97. ACM press, 1997. https://doi.org/10.1145/1120212.1120376 
[2] Alan F. Blackwell and Thomas R.G. Hollender. A cognitive dimensions questionnaire optimised for users. In Proceedings of 12th Workshop of the Psychology of Programming Interest Group, 2000.

[3] Anna E. Bobkowska. Cognitive dimensions questionnaire applied to visual modelling language evaluation - a case study1. In Proceedings of Joint Conf. EASE \& PPIG. M. Petre \& D. Budgen (Eds), 2003.

[4] J. Bonnie and K. David. Using goms for user interface design and evaluation: Which technique? ACM Transactions on Computer-Human Interaction, 4(3), 1996.

[5] J. Bonnie and Kieras. David. The goms family of user interface analysis techniques: Comparison and contrast. ACM Transactions on Computer-Human Interaction, 4(3), 1996. https://doi.org/10.1145/235833.236054

[6] W. Buxton and B. Myers. A study in two-handed input. In Proceedings of the CHI '86 Conference on Human Factors in Computing Systems. ACM, 1986. https://doi.org/10. $\underline{1145 / 22627.22390}$

[7] K. Cox. Cognitive dimensions of use cases -feedback from a learner questionnaire. In Proceedings of 12th Workshop of the Psychology of Programming Interest Group, Cozenza Italy, April 2000.

[8] J. Du, B. Havard, and H. Li. Dynamic online discussion:task-oriented interaction for deep learning. Educational Media International, 42(3), 2005.https://doi.org/10.1080/ 09523980500161221

[9] P. M. Fitts. The information capacity of the human motor system in controlling the amplitude of movement. Journal of Experimental Psychology, 47(6), 1954.

[10] F.Jill, T.Dana, and B.Cyd. An exploratory study of online learning for professional development: Should club managers go the distance. Merlot Journal of Online Learning and Teaching, 4(3), 2008.

[11] G. Golovchinsky. Cognitive dimensions analysis of interfaces for information seeking. In Proceedings of HCIR 09 workshop, 2009.

[12] Nina Hollender, Cristian Hofmann, Michael Deneke, Bernhard Schmitz, Review: Integrating cognitive load theory and concepts of human-computer interaction, Computers in Human Behavior, v.26 n.6, p.1278-1288, November, 2010.https://doi.org/10.1016/ j.chb.2010.05.031

[13] P. Kabbash, I. MacKenzie, and W. Buxton. Human performance using computer input devices in the preferred and non-preferred hands. In Proceedings of CHI'93, Human Factors in Computing Systems, 1993. https://doi.org/10.1145/169059.169414

[14] I.MacKenzie. Fitts' law as a performance model in human-computer interaction, 1999.

[15] B Bahli. R. Saade. The impact of cognitive absorption on perceived usefulness and perceived ease of use in on-line learning: an extension of the, technology acceptance model. Information and Management, 42, 2005. https://doi.org/10.1016/j.im.2003.12.013

[16] T C. Reeves and W. D. Aggen . Enhancing e-learning assessment and evaluation strategies. In Proceedings of ELEARN 2002 Vol. 2002 (1), 806811, 2002.

[17] C Stuar, M. Thomas, and A. Newell. The keystroke-level model for user performance with interactive systems. Communications of the ACM, 23(6), 1980.

[18] P Viviani and C. Terzuolo. Trajectory determines movement dynamics. Neuroscience, 1982. https://doi.org/10.1016/0306-4522(82)90277-9

[19] P Zaharias. Developing a usability evaluation method for e-learning applications: From functional usability to motivation to learn, 2008.

[20] P Zhang, N. Li, and H. Sun. Affective quality and cognitive absorption extending technology acceptance research. In Proceedings of the Hawaii International Conference on System Sciences, January 2006. 
[21] O Belloa, S., Zeadallyb, 'Toward efficient smartification of the Internet of Things (IoT) services', Future Generation Computer Systems, In Press, 2017, DOI https://doi.org/10. $\underline{1016 / \text { i.future.2017.09.083 }}$

\section{Authors}

Henda Chorfi Ouertani is currently Assistant Professor at the Information Technology Department, College of Computer and Information Sciences, King Saud University (KSU), Riyadh, KSA. Before joining KSU, she was head of the Computer Science Department at ISTMT and member of the Research Laboratory LaTICE, (University of Tunis El Manar, Tunisia). Her Research projects Involvement are elearning tools and environments, Technology-Enhanced Learning, Adaptive/Adaptable systems and user modeling. She serves on program committees and organization committees of numerous international conferences.

Ghada Al-Hudhud: This author received her $\mathrm{PhD}$ in the field of controlling multiple mobile cooperative robots from De Montfort University 2005, Leicester, UK. She served as a researcher and lecturer at the same university 2004-2006. She served then at Alahliyya Amman University, Amman, Jordan as a Head of Software Engineering Department 2006-2010. Currently, she serves as full professor at Department of Information Technology, College of Computer and Information Sciences, King Saud University, Riyadh, KSA. She has several publications in the field of brain computing in the areas of personal identification, multimodal command control systems, and smart office control. Dr. Al-Hudhud is a professional member in British Computer Society BCS, Association of Computing Machinery. She has earned the best researcher award from Prince Sultan Univesity 6 years ago.

Article submitted 2019-01-08. Resubmitted 2019-02-17. Final acceptance 2019-02-28. Final version published as submitted by the authors. 